6. Orlando G, Soker S, Wood K. Operational tolerance after liver transplantation. J Hepatol 2009; 50 : 1247-57.

7. Berlanda M, Di Cocco P, Mazzotta C, et al. Clinical operational tolerance after kidney transplantation: a short literature review. Transplant Proc 2008; 40 : 1847-51.

8. Roussey-Kesler G, Giral M, Moreau A, et al. Clinical operational tolerance after kidney transplantation. Am J Transplant 2006; 6: 736-46.

9. Sawitzki B, Pascher A, Babel N, et al. Can we use biomarkers and functional assays to implement personalized therapies in transplantation? Transplantation 2009; 87 : 1595-601.

10. Bunnag $S$, Einecke G, Reeve J, et al. Molecular correlates of renal function in kidney transplant biopsies. J Am Soc Nephrol 2009; 20 : 1149-60.

11. Ashton-Chess J, Giral M, Soulillou JP, et al. Using biomarkers of tolerance and rejection to identify high- and low-risk patients following kidney transplantation. Transplantation 2009; 87 : 595-9.

12. Mengel $M$, Reeve J, Bunnag $S$, et al. Scoring total inflammation is superior to the current Banff inflammation score in predicting outcome and the degree of molecular disturbance in renal allografts. Am J Transplant 2009; 9: 1859-67.

13. Anglicheau D, Suthanthiran M. Noninvasive prediction of organ graft rejection and outcome using gene expression patterns. Transplantation 2008; $86: 192-9$

14. Brouard S, Mansfield $\varepsilon$, Braud C, et al. Identification of a peripheral blood transcriptional biomarker panel associated with operational renal allograft tolerance. Proc Natl Acad Sci USA 2007 ; 104 : 15448-53.

15. Newell KA, Asare A, Kirk AD, et al. Identification of a $B$ cell signature associated with renal transplant tolerance in humans. J Clin Invest 2010; 120 : 1836-47.

16. Sagoo P, Perucha $\varepsilon$, Sawitzki B, et al. Development of a cross-platform biomarker signature to detect renal transplant tolerance in humans. J Clin Invest 2010; 120 : 1848-61.

17. Pallier A, Hillion S, Danger $R$, et al. Patients with drug-free long-term graft function display increased numbers of peripheral $B$ cells with a memory and inhibitory phenotype. Kidney Int 2010 ; 78 : 503-13.

18. Louis S, Braudeau C, Giral M, et al. Contrasting CD25hiCD4+T cells/FOXP3 patterns in chronic rejection and operational drug-free tolerance. Transplantation $2006 ; 81: 398-407$.

19. Yanaba K, Bouaziz JD, Haas KM, et al. A regulatory $B$ cell subset with a unique CDIdhiCD5+ phenotype controls T cell-dependent inflammatory responses. Immunity 2008; 28 : 639-50.

20. Hauer J, Puschner S, Ramakrishnan P, et al. TNF receptor (TNFR)-associated factor (TRAF) 3 serves as an inhibitor of TRAF2/5-mediated activation of the noncanonical NF-kappaB pathway by TRAF-binding TNFRs. Proc Natl Acad Sci USA 2005 ; 102 : 2874-9.

21. Einecke $G$, Reeve J, Sis B, et al. A molecular classifier for predicting future graft loss in late kidney transplant biopsies. J Clin Invest 2010; 120 : 1862-72.

22. Ashton-Chess J, Giral M, Mengel M, et al. Tribbles-1 as a novel biomarker of chronic antibody-mediated rejection. J Am Soc Nephrol 2008 ; 19 : 1116-27.

23. Suthanthiran M. Human renal allograft rejection: molecular characterization. Nephrol Dial Transplant 1998; 13 Suppl 1: 21-4.

24. Foucher $Y$, Daguin P, Akl A, et al. A clinical score highly predictive of long-term kidney graft survival. Kidney Int 2010 ; sous presse.
NOUVELL

\section{Leucémie myéloïde chronique et thérapeutiques ciblées}

Bientôt l'embarras du choix?

Gabriel Etienne, Françoise Huguet
Service d'hémato-oncologie,

Institut Bergonié, Centre de lutte contre

le cancer de Bordeaux et du Sud-Ouest,

229 cours de l'Argonne,

33076 Bordeaux Cedex, France.

etienne@bergonie.org

Service d'hématologie, CHU Purpan,

Place du Dr Baylac,

31059 Toulouse Cedex, France.

huguet.f@chu-toulouse.fr

tinib, dont l'effet anti-leucémique est plus marqué in vitro que celui de l'imatinib, vont rapidement s'imposer comme une option thérapeutique majeure chez les patients résistants ou intolérants à l'imatinib [5, 6]. L'absence d'intolérance croisée entre ces deux molécules et l'imatinib, leur efficacité en cas de mutation du domaine tyrosine kinase d'ABL - principale cause de résistance à l'imatinib identifiée à ce jour [11] - rendent compte de leur efficacité dans ces situations.

La question de l'intérêt de ces deux molécules dans le traitement de la maladie, et ce dès le diagnostic, va être rapidement soulevée par les premiers résultats des essais de phase II, montrant des taux et une rapidité de réponse, tant sur le plan cytogénétique que moléculaire, largement supérieurs à ceux historiquement observés avec l'imatinib [7-9]. La comparaison prospective et randomisée s'imposait.

\section{La suprématie}

Situation presque inédite dans une maladie modèle, les résultats de deux de phase III menés par l'industrie pharof Medecine [1, 2]. Ils comparent respectivement l'efficacité du nilotinib et sine kinase, à celle de l'imatinib chez des patients présentant une leucémie myéloïde chronique en phase chronique (LMC-PC) au diagnostic (voir Encadré) [10]. Bien qu'inscrit dans une certaine logique de développement industriel, le défi était de taille et la question posée en apparence simple: peut-on faire mieux que l'imatinib (voir Encadré)? $\varepsilon n$ effet, les résultats de l'étude IRIS (international randomised study of interrapport avec la maladie) [4].

Dès 2004, d'autres inhibiteurs oraux de l'activité tyrosine kinase d'ABL vont émerger. Parmi ceux-ci, le nilotinib et le dasa- 


\section{L'imatinib, le premier inhibiteur de la kinase Abl}

« La leucémie myéloïde chronique (LMC) est une affection maligne clonale de la cellule souche hématopoïétique caractérisée cliniquement par une évolution inéluctable vers une phase de leucémie aiguë, et biologiquement par la présence de l'oncogène $B C R-A B L$ (breakpoint cluster region-Abelson) [correspondant au chromosome Philadelphie, une translocation équilibrée entre les chromosomes 9 et 22] dans toutes les cellules leucémiques. Au cours de la phase aiguë (blastique) de la maladie, l'expression de BCR-ABL dans les cellules leucémiques augmente progressivement, cause probable d'événements génétiques secondaires responsables d'une instabilité génétique qui pourrait être à l'origine de la chimiorésistance observée au cours de cette phase. [...] En 1996, un inhibiteur des molécules à activité tyrosine kinase (TK) a été identifié, conduisant à la synthèse d'une molécule inhibant l'activité [tyrosine-kinase] induite par le PDGF (platelet-derived growth factor) mais aussi celle de v-abl et de BCR-ABL. Cette molécule, un dérivé de la 2-aminopyrimidine, agit au niveau de la poche à ATP de la portion $A B L$ de $B C R-A B L$ de manière sélective alors qu'elle n'a aucun effet inhibiteur sur l'activité TK d'autres récepteurs comme le VEGF-R (vascular endothelial growth factor receptor) et $\varepsilon G F-R$ (epidermal growth factor receptor). Cette sélectivité a conduit rapidement à l'introduction de cet inhibiteur (initialement appelé CGP57148, STI571 puis l'imatinib mésylate) en clinique, et depuis 1998, plusieurs milliers de patients atteints de LMC ont été traités avec cette molécule ».

(reproduit de [10])

Deux essais cliniques internationaux nous livrent leurs premiers résultats.

\section{Critères d'évaluation de l'efficacité des nouveaux inhibiteurs de l'activité tyrosine kinase}

\section{Imatinib versus Nilotinib}

L'objectif de l'essai était de comparer l'efficacité de l'imatinib (400 mg/j) à celle du nilotinib (administré aux posologies de $600 \mathrm{mg} / \mathrm{j}$ ou $800 \mathrm{mg} / \mathrm{j}$ ) chez des patients présentant une LMC-PC au diagnostic. Le critère principal de jugement était le taux de réponse moléculaire majeure, définie par une diminution supérieure ou égale à 3 log du taux de transcrits $B C R-A B L$, au terme de la première année de traitement. L'étude a inclus 846 patients. Les caractéristiques des patients et les principaux résultats sont présentés dans le Tableau IA.

\section{Imatinib versus Dasatinib}

L'objectif de l'essai était de comparer l'efficacité de l'imatinib (400 mg/j) au dasatinib à la posologie de $100 \mathrm{mg} / \mathrm{j}$ chez des patients présentant une LMC$P C$ au diagnostic. Le critère principal de jugement était le taux de réponse cytogénétique complète confirmée, définie par l'absence de détection du chromosome Philadephie à l'examen cytogénétique des métaphases de cellules médullaires à 28 jours d'intervalle, au terme de la première année de traitement. L'étude a inclus 519 patients. Les caractéristiques des patients et les principaux résultats sont présentés dans le Tableau IB. Avec un an de suivi minimum pour la totalité des patients, ces deux études montrent la supériorité des inhibiteurs de tyrosine kinase de «deuxième génération » nilotinib et dasatinib sur l'imatinib tant en termes de réponse cytogénétique complète que de réponse moléculaire majeure dès la fin de la première année de traitement, et ce pour tous les sous-groupes de patients définis par les scores de Sokal ou de Hasford. Le risque de progression vers les phases accélérée ou blastique de la maladie apparaît également inférieur chez les patients traités par nilotinib et dasatinib. Par ailleurs, aucune progression de la maladie n'a été observée chez les patients ayant obtenu une réponse moléculaire majeure sous traitement.

Si l'efficacité de ces deux molécules semble proche au vu de ces résultats préliminaires, leur profil de tolérance diffère quelque peu. Ainsi la toxicité hématologique apparaît plus fréquente et plus marquée chez les patients traités par dasatinib, les éruptions cutanées et les perturbations des enzymes hépatiques et pancréatiques plus fréquentes chez les patients traités par nilotinib, la posologie de $600 \mathrm{mg} / \mathrm{j}$ étant globalement mieux supportée. L'incidence et la gravité des épanchements pleuraux sous dasatinib apparaissent, avec un an de recul, inférieures aux données précédemment rapportées [9].

\section{Et maintenant,} quelle démarche thérapeutique? Au-delà des différences méthodologiques et de présentation des résultats entre ces deux essais, la supériorité démontrée des inhibiteurs de tyrosine kinase de deuxième génération sur l'imatinib pose dans cette indication plusieurs questions de fond:

- la rapidité d'efficacité de ces nouvelles molécules se traduira-t-elle par un bénéfice durable pour les patients? Le suivi médian pour l'instant court le suggère, montrant un taux de progression vers les phases avancées de la maladie inférieur à celui observé sous imatinib et ce dès la fin de la première année de traitement;

- quels seront, à efficacité équivalente, les déterminants du choix de l'une ou l'autre de ces molécules si leur supériorité sur l'imatinib se confirme et valide leur utilisation dans cette indication? Nul doute que leur profil de tolérance respectif et leur coût pour la collectivité seront des arguments de poids.

L'avenir proche ne manquera pas de répondre à ces questions.

Après dix ans d'un succès thérapeutique inégalé, la suprématie de l'imatinib est remise en cause. Au-delà d'une compétition industrielle intense, ces deux essais thérapeutiques internationaux menés en parallèle confirment l'intérêt toujours vif porté à une maladie rare mais néanmoins modèle. $\diamond$

Chronic myeloid leukemia

and targeted therapies:

too many choices? 
Tableau I. Résultats des essais cliniques comparant le nilotinib et le dasatinib à l'imatinib chez des patients présentant une leucémie myéloïde chronique en phase chronique au diagnostic : patients et résultats au terme de la première année de traitement (d'après [1, 2]).

\begin{tabular}{|c|c|c|c|c|c|}
\hline A & & $\begin{array}{l}\text { Nilotinib } \\
600 \mathrm{mg} / \mathrm{j}\end{array}$ & $\begin{array}{l}\text { Nilotinib } \\
800 \mathrm{mg} / \mathrm{j}\end{array}$ & $\begin{array}{l}\text { Imatinib } \\
400 \mathrm{mg} / \mathrm{j}\end{array}$ & p-value* \\
\hline \multirow{4}{*}{$\begin{array}{c}\text { Population : } \\
\text { caractéristiques } \\
\text { à l'inclusion }\end{array}$} & Effectif & $n=281$ & $n=282$ & $n=283$ & \\
\hline & Âge moyen - (intervalle) & $47(18-85)$ & $47(18-81)$ & $46(18-80)$ & \\
\hline & $\begin{array}{l}\text { Score de Sokal - risque faible/ } \\
\text { intermédiaire/élevé (\%) }\end{array}$ & $37 / 36 / 28$ & $37 / 36 / 28$ & $37 / 36 / 28$ & \\
\hline & $\begin{array}{l}\text { Anomalies cytogénétiques additionnelles au } \\
\text { chromosome Philadelphie - } \mathrm{n}(\%)\end{array}$ & $34(12)$ & $44(16)$ & $31(11)$ & \\
\hline \multirow{6}{*}{ Efficacité } & Réponse moléculaire majeure au mois 6 (\%) & 33 & 30 & 12 & \\
\hline & Réponse moléculaire majeure au mois 12 (\%) & 44 & 43 & 22 & $<0,001$ \\
\hline & Réponse cytogénétique complète au mois 6 (\%) & 67 & 63 & 45 & \\
\hline & Réponse cytogénétique complète au mois 12 (\%) & 80 & 78 & 65 & $<0,001$ \\
\hline & Progression vers phases avancées au cours & $<1$ & $<1$ & 4 & 0,01 \\
\hline & de la $1^{\text {re }}$ année (\%) & & & & 0,004 \\
\hline \multirow{3}{*}{ Tolérance } & $\begin{array}{l}\text { Réduction de posologie et interruption temporaire } \\
\text { de traitement pour effet secondaire (\%) }\end{array}$ & 59 & 66 & 52 & \\
\hline & $\begin{array}{l}\text { Durée cumulative moyenne d'arrêt de traitement pour } \\
\text { effet secondaire (jours) }\end{array}$ & 19 & 22 & 15 & \\
\hline & Arrêt définitif de traitement pour effet secondaire (\%) & 5 & 9 & 7 & \\
\hline
\end{tabular}

A. Nilotinib 600 et $800 \mathrm{mg} / \mathrm{j}$ versus imatinib $400 \mathrm{mg} / \mathrm{j}$ : patients et résultats de la première année de traitement. * Comparaison nilotinib $800 \mathrm{mg} / \mathrm{j}$ versus imatinib $400 \mathrm{mg} / \mathrm{j}$ et nilotinib $600 \mathrm{mg} / \mathrm{j}$ versus imatinib $400 \mathrm{mg} / \mathrm{j}$.

\begin{tabular}{|c|c|c|c|c|}
\hline B & & $\begin{array}{l}\text { Dasatinib } \\
100 \mathrm{mg} / \mathrm{j}\end{array}$ & $\begin{array}{l}\text { Imatinib } \\
400 \mathrm{mg} / \mathrm{j}\end{array}$ & p-value \\
\hline \multirow{4}{*}{$\begin{array}{c}\text { Population : } \\
\text { caractéristiques } \\
\text { à l'inclusion }\end{array}$} & Effectif & $n=259$ & $n=260$ & \\
\hline & Âge moyen - (intervalle) & $46(18-84)$ & $49(18-78)$ & \\
\hline & $\begin{array}{l}\text { Score de Hasford - risque faible/ } \\
\text { intermédiaire/élevé (\%) }\end{array}$ & $33 / 48 / 19$ & $33 / 47 / 19$ & \\
\hline & Blastes médullaires moy \% (intervalle) & $2(0,0-14)$ & $2(0,0-12)$ & \\
\hline \multirow{5}{*}{ Efficacité } & Réponse moléculaire majeure au mois 6 (\%) & 27 & 8 & \\
\hline & Réponse moléculaire majeure au mois $12(\%)$ & 46 & 28 & $<0,0001$ \\
\hline & Réponse cytogénétique complète au mois 6 (\%) & 73 & 59 & \\
\hline & Réponse cytogénétique complète confirmée au mois 12 (\%) & 77 & 66 & 0,007 \\
\hline & Progression vers phases avancées au cours de la $1^{\text {re }}$ année (\%) & 1,9 & 3,5 & \\
\hline \multirow{3}{*}{ Tolérance } & Dose moyenne délivrée - mg/j (intervalle) & $99(21-136)$ & $400(125-657)$ & \\
\hline & Traitement poursuivi pendant durée étude (\%) & 84 & 81 & \\
\hline & Arrêt définitif de traitement pour effet secondaire (\%) & 5 & 4 & \\
\hline
\end{tabular}

B. Dasatinib $100 \mathrm{mg} / \mathrm{j}$ versus imatinib $400 \mathrm{mg} / \mathrm{j}$ : patients et résultats de la première année de traitement. 
CONFLIT D'INTÉRÊTS

les auteurs déclarent n'avoir aucun conflit d'intérêt concernant les données publiées dans cet article.

\section{RÉFÉRENCES}

1. Saglio G, Kim DW, Issaragrisil S, et al. Nilotinib versus imatinib for newly diagnosed chronic myeloid leukemia. N Engl J Med 2010 ; 362 : 2251-9.

2. Kantarjian H, Shah NP, Hocchaus A, et al. Dasatinib versus imatinib in newly diagnosed chronic-phase chronic myeloid leukemia. $N$ Engl J Med 2010 ; $362: 2260-70$.

3. O'Brien SG, Guilhot F, Larson RA, et al. Imatinib compared with interferon and low-dose cytarabine for newly diagnosed chronic-phase chronic myeloid leukemia. N Engl J Med 2003; 348 : 994-1004.

4. Hochhaus A, O'Brien SG, Guilhot F, et al. Six-year follow-up of patients receiving imatinib for the firstline treatment of chronic myeloid leukemia. Leukemia $2009 ; 23$ : 1054-61.

5. Kantarjian $\mathrm{H}$, Giles F, Wunderle L, et al. Nilotinib in imatinib-resistant CML and Philadelphia chromosome-positive ALL. N EnglJ Med 2006 ; $354: 2542-51$.

6. Talpaz M, Shah NP, Kantarjian H, et al. Dasatinib in imatinib-resistant Philadelphia chromosome-positive leukemias. N Engl J Med 2006 ; 354 : 2531-41.

7. Rosti G, Palandri F, Castagnetti F, et al. Nilotinib for the frontline treatment of $\mathrm{Ph}+$ chronic myeloid leukemia. Blood 2009; 114 : 4933-8.
NOUVELLE

\section{Une souris peut-elle fabriquer un pancréas d'éléphant?}

Laure Coulombel
8. Cortes JE, Jones D, O'Brien S, et al. Nilotinib as frontline treatment for patients with chronic myeloid leukemia in early chronic phase. J Clin Oncol 2010; $28: 392-7$.

9. Cortes JE, Jones D, O'Brien S, et al. Results of dasatinib therapy in patients with early chronicphase chronic myeloid leukemia.J Clin Oncol 2010; $28: 398-404$.

10. Turhan A. Imatinib mesylate: a major breakthrough in the treatment of chronic myelogenous leukemia. Med Sci (Paris) 2003; $19: 667-8$

11. Roche-Lestienne C, Mahon FX, Preudhomme C. Origin of resistance to Imatinib mesylate: lessons learned from this experience. Med Sci (Paris) 2004 ; $20: 1125-30$
Médecine/Sciences, ADR Inserm Paris 5, 2, rue d'Alésia, 75014 Paris, France. laure.coulombel@inserm.fr

de même que la niche mésodermique, vide chez les animaux $p d x 1^{-/-}$, mais qui conserve la capacité de guider la spécification de cellules $\varepsilon$ S ou iPS $p d x 1^{+/+}$avec lesquelles elles entreraient en contact. De fait, des IPS ou des ES murines (avec une étiquette GFP) $p d x 1^{+/+}$injectées dans des blastocystes $p d x 1^{-/-}$ensuite transférés dans l'utérus de souris femelles pseudogestantes contribuent à la formation d'embryons viables (25\%-40\%). Chez ces derniers, tous les tissus sont chimériques (taux avoisinant $50 \%$ ) sauf le placenta, ce qui était attendu. Plus important, ces souris chimériques ont développé un pancréas complet fonctionnel dont les structures endocrine et exocrine, normales, sont exclusivement d'origine donneur ( $\varepsilon S$ ou iPS $p d x 1^{+/+}$) alors que l'environnement stromal est chimérique. Les îlots $\beta$ pancréatiques issus de ces iPS ont pu être greffés avec succès à des receveurs diabétiques, confirmant ce qu'avait démontré la complémentation des embryons tétraploïdes, la capacité des iPS à former un organe entier in vivo [1].

La même démarche a ensuite été appliquée en situation xénogénique: des cellules iPS et ES de rat et de souris ont été 